\title{
Critical Doping for the Onset of Fermi-Surface Reconstruction by Charge-Density-Wave Order in the Cuprate Superconductor $\mathrm{La}_{2-x} \mathrm{Sr}_{x} \mathrm{CuO}_{4}$
}

\author{
S. Badoux, ${ }^{1, *}$ S. A. A. Afshar, ${ }^{1}$ B. Michon, ${ }^{1}$ A. Ouellet, ${ }^{1}$ S. Fortier, ${ }^{1}$ D. LeBoeuf, ${ }^{2}$ T. P. Croft, ${ }^{3}$ C. Lester, ${ }^{3}$ \\ S. M. Hayden, ${ }^{3}$ H. Takagi, ${ }^{4}$ K. Yamada ${ }^{5}$ D. Graf, ${ }^{6}$ N. Doiron-Leyraud, ${ }^{1}$ and Louis Taillefer ${ }^{1,7, t}$ \\ ${ }^{1}$ Département de Physique \& RQMP, Université de Sherbrooke, Sherbrooke, Québec J1K 2R1, Canada \\ ${ }^{2}$ Laboratoire National des Champs Magnétiques Intenses, UPR 3228, (CNRS-INSA-UJF-UPS), \\ Grenoble 38042, France \\ ${ }^{3}$ H. H. Wills Physics Laboratory, University of Bristol, Bristol BS8 1TL, United Kingdom \\ ${ }^{4}$ Department of Advanced Materials, University of Tokyo, Kashiwa 277-8561, Japan \\ ${ }^{5}$ Institute of Materials Structure Science, High Energy Accelerator Research Organization \& The \\ Graduate University for Advanced Studies, Oho, Tsukuba 305-0801, Japan \\ ${ }^{6}$ National High Magnetic Field Laboratory, Tallahassee, Florida 32310, USA \\ ${ }^{7}$ Canadian Institute for Advanced Research, Toronto, Ontario M5G 1Z8, Canada \\ (Received 18 November 2015; revised manuscript received 29 February 2016; published 6 April 2016)
}

\begin{abstract}
The Seebeck coefficient $S$ of the cuprate superconductor $\mathrm{La}_{2-x} \mathrm{Sr}_{x} \mathrm{CuO}_{4}$ (LSCO) was measured in magnetic fields large enough to access the normal state at low temperatures, for a range of $\mathrm{Sr}$ concentrations from $x=0.07$ to $x=0.15$. For $x=0.11,0.12,0.125$, and $0.13, S / T$ decreases upon cooling to become negative at low temperatures. The same behavior is observed in the Hall coefficient $R_{\mathrm{H}}(T)$. In analogy with other hole-doped cuprates at similar hole concentrations $p$, the negative $S$ and $R_{\mathrm{H}}$ show that the Fermi surface of LSCO undergoes a reconstruction caused by the onset of charge-density-wave modulations. Such modulations have indeed been detected in LSCO by x-ray diffraction in precisely the same doping range. Our data show that in LSCO this Fermi-surface reconstruction is confined to $0.085<p<0.15$. We argue that in the field-induced normal state of LSCO, charge-density-wave order ends at a critical doping $p_{\mathrm{CDW}}=0.15 \pm 0.005$, well below the pseudogap critical doping $p^{\star} \simeq 0.19$.

DOI: 10.1103/PhysRevX.6.021004

Subject Areas: Condensed Matter Physics,

Materials Science, Superconductivity
\end{abstract}

\section{INTRODUCTION}

Since the discovery of quantum oscillations [1] and a negative Hall coefficient $R_{\mathrm{H}}$ [2] in the cuprate superconductor $\mathrm{YBa}_{2} \mathrm{Cu}_{3} \mathrm{O}_{y}$ (YBCO), it has become clear that the Fermi surface of underdoped YBCO undergoes a reconstruction at low temperatures that produces a small electron pocket [3], in a doping range from $p=0.08$ to $p \simeq 0.15$ [4]. This Fermi-surface reconstruction (FSR) was also detected as a sign change in the Seebeck coefficient $S(T)$, going from positive at high temperatures to negative at low temperatures [5]. A strikingly similar change of sign in $S(T)$ observed in the cuprate $\mathrm{La}_{1.8-x} \mathrm{Eu}_{0.2} \mathrm{Sr}_{x} \mathrm{CuO}_{4}$ (EuLSCO) [6] suggested that the stripe order known to exist in Eu-LSCO [7]—a combination of charge-density-wave (CDW) and spin-density-wave (SDW) modulations-is responsible for the FSR in both materials. The observation

\footnotetext{
*sven.badoux@usherbrooke.ca

†louis.taillefer@usherbrooke.ca
}

Published by the American Physical Society under the terms of the Creative Commons Attribution 3.0 License. Further distribution of this work must maintain attribution to the author $(s)$ and the published article's title, journal citation, and DOI. of CDW modulations in YBCO by NMR [8] and x-ray diffraction (XRD) $[9,10]$ confirmed this conjecture and demonstrated that it is the CDW (and not the SDW) modulations that cause the FSR.

In YBCO, the drop in $R_{\mathrm{H}}(T)$ and $S / T$ begins at a temperature $T_{\max }$ that peaks at $p=0.12$ [Fig. 1(a)]. The drop is attributed to the CDW modulations detected by XRD [11,12] and NMR [21] below a temperature $T_{\mathrm{CDW}}$ in the same doping range as the FSR [4], with $T_{\mathrm{CDW}}$ also peaking at $p=0.12$ [Fig. 1(a)].

In $\mathrm{HgBa}_{2} \mathrm{CuO}_{4+\delta}(\mathrm{Hg} 1201)$, high-field measurements of Hall and Seebeck coefficients revealed a similar FSR [22], confirmed by the observation of quantum oscillations [23] and again attributed to XRD-detected CDW modulations [24]. All this suggests that CDW modulations and the associated FSR are generic properties of hole-doped cuprates in the vicinity of $p=0.12$. A major outstanding question is the following: $\mathrm{Up}$ to what critical doping $p_{\mathrm{CDW}}$ do CDW modulations extend in the phase diagram (Fig. 1), in particular, in the field-induced normal state at $T=0$ ? In this context, the material LSCO offers a powerful platform since good crystals can be grown with $p$ up to 0.3 and beyond. CDW modulations have been observed in LSCO with XRD, at $p \simeq 0.12[13,14,25]$, but there is little information about the associated FSR. 

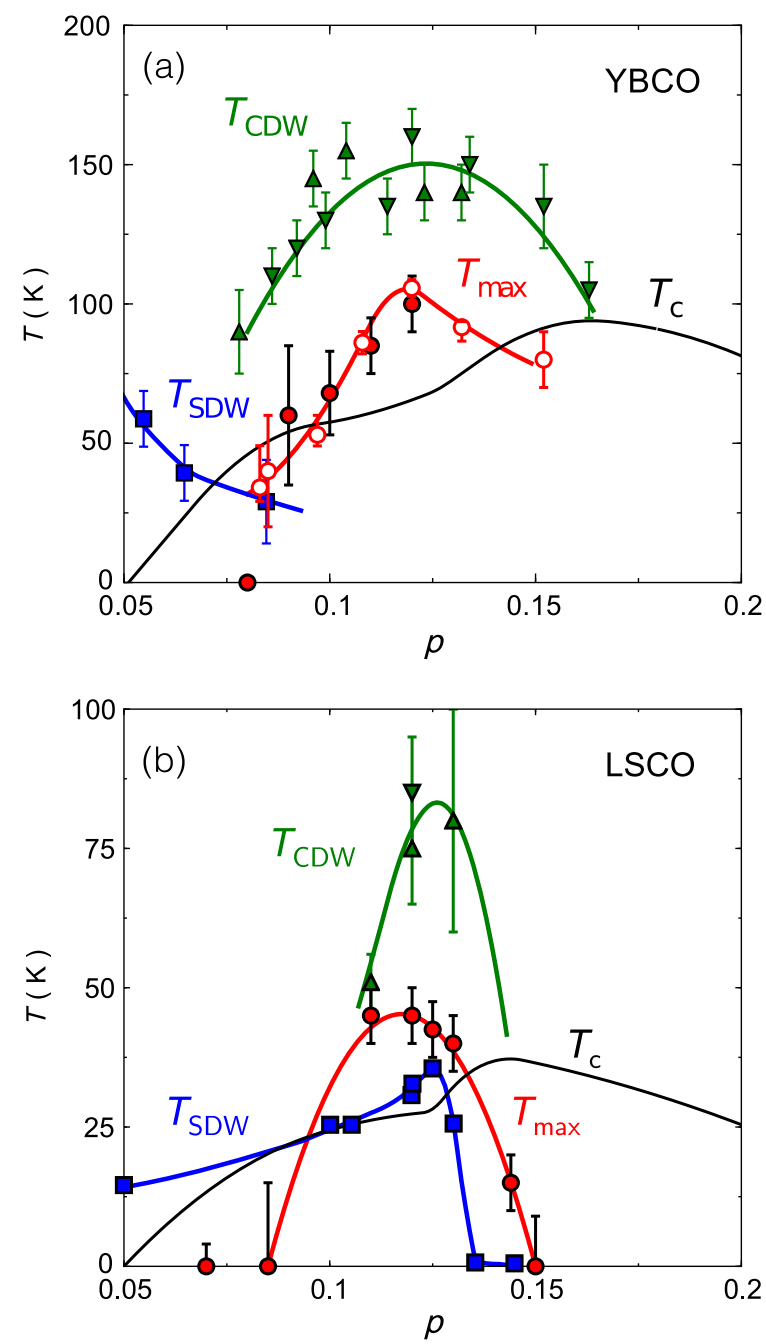

FIG. 1. Temperature-doping phase diagram of the cuprate superconductors YBCO (a) and LSCO (b). The superconducting transition temperature $T_{\mathrm{c}}$ is drawn as a black line. CDW modulations are detected by $\mathrm{x}$-ray diffraction below $T_{\mathrm{CDW}}$ (green triangles) in YBCO (up triangles [11], down triangles [12]) and LSCO (up triangles [13], down triangle [14]). SDW modulations are detected by neutron diffraction below $T_{\mathrm{SDW}}$ (blue squares) in YBCO [15] and LSCO [16-20]. When plotted as $S / T$ vs $T$, the normal-state Seebeck coefficient peaks at a temperature $T_{\max }$ (full red circles) before it drops at low temperatures because of Fermisurface reconstruction (YBCO, Ref. [6]; LSCO, this work, Figs. 3 and 4). A similar $T_{\max }$ can also be defined for the Hall coefficient (open red circles), below which $R_{\mathrm{H}}(T)$ drops at low temperatures (YBCO, Ref. [4]).

In this paper, we report high-field measurements of the Seebeck coefficient in LSCO single crystals at several dopings, which show that $S$ becomes negative in the normal state at low temperatures in precisely the doping range where CDW modulations are detected by XRD. $R_{\mathrm{H}}$ is also found to be negative in that range. The FSR in LSCO is therefore very similar to the FSR in YBCO and Hg1201. Our data show that the FSR does not extend above $p=0.15$, strong evidence that CDW order in LSCO ends at a critical doping $p_{\mathrm{CDW}}=0.15$. This implies that in the normal state of LSCO, the phase of CDW order ends well before the pseudogap phase, which ends at the critical doping $p^{\star} \simeq 0.19$ (Ref. [26]).

\section{METHODS}

Single crystals of LSCO were grown by the flux-zone technique with $\mathrm{Sr}$ concentrations $x=0.085,0.11,0.12$, and 0.13 at the University of Bristol, $x=0.07$ and 0.125 at the University of Tokyo, and $x=0.144$ and 0.15 at Tohoku University. Samples were cut in the shape of rectangular platelets, with typical dimensions $0.5 \mathrm{~mm} \times 1.0 \mathrm{~mm} \times$ $0.1 \mathrm{~mm}$. The hole concentration (doping) $p$ is taken to be $p=x$. The (zero-resistance) superconducting transition temperature of the eight samples is $T_{\mathrm{c}}=12.7,20.2,26.2$, $27.5,28.0,32.3,37.2$, and $36.5 \mathrm{~K}$ for $p=0.07,0.085$, $0.11,0.12,0.125,0.13,0.144$, and 0.15 , respectively. The Seebeck coefficient was measured, as described elsewhere [6], at Sherbrooke (all samples) up to $H=20 \mathrm{~T}$, at the National High Magnetic Field Laboratory (NHMFL) in Tallahassee up to $H=34 \mathrm{~T}(x=0.125$ and 0.15$)$ and up to $H=45 \mathrm{~T}(x=0.13)$, and at the Laboratoire National des Champs Magnétiques Intenses (LNCMI) in Grenoble up to $H=34 \mathrm{~T}(x=0.07$ and 0.144$)$. The Hall coefficient of samples with $x=0.11,0.12,0.125$, and 0.13 was measured, as described elsewhere [4], at Sherbrooke in $H=16 \mathrm{~T}$. All crystals have an orthorhombic crystal structure, and they are twinned. The thermal gradient or electrical current was applied in the basal plane, while the magnetic field was applied along the $c$ axis.

\section{SEEBECK COEFFICIENT}

In Fig. 2, the Seebeck data for six samples are plotted as $S / T$ vs $H$ for several temperatures. We see that for $x=$ 0.125 [Fig. 2(c)] and $x=0.13$ [Fig. 2(d)], $S$ becomes negative at high fields and low temperatures. This shows that a negative $S$ is a property of the normal state of LSCO at these dopings, as in YBCO, Eu-LSCO, and Hg1201. At $x=0.144$, we see that at high fields, $S / T$ decreases when the temperature drops below $T=15 \mathrm{~K}$ [Fig. 2(e)]. In contrast, no such decrease is observed at $x=0.15$, down to the lowest temperature [Fig. 2(f)]. At $x=0.07, S / T$ increases steadily with decreasing $T$ at high fields, down to the lowest temperature [Fig. 2(a)]. This is also true at $x=$ 0.085 [Fig. 2(b)]. Although here our data only go to $20 \mathrm{~T}$, the crossing of the lowest isotherms shows that $S / T$ keeps increasing down to $T=15 \mathrm{~K}$, at least.

In Figs. 3 and 4, we plot $S / T$ vs $T$, at high fields. In Fig. 3(a), we see that the drop in $S / T$ at $x=0.125$ to negative values starts below a temperature $T_{\max } \simeq 40 \mathrm{~K}$. This is also the case at $x=0.11$ and 0.13 [Fig. 4(a)]. In Fig. 4(b), we compare data on three samples taken in identical conditions, at $H=16 \mathrm{~T}$. [Although the LSCO sample with $x=0.12$ was only measured up to $18 \mathrm{~T}, S / T$ at 

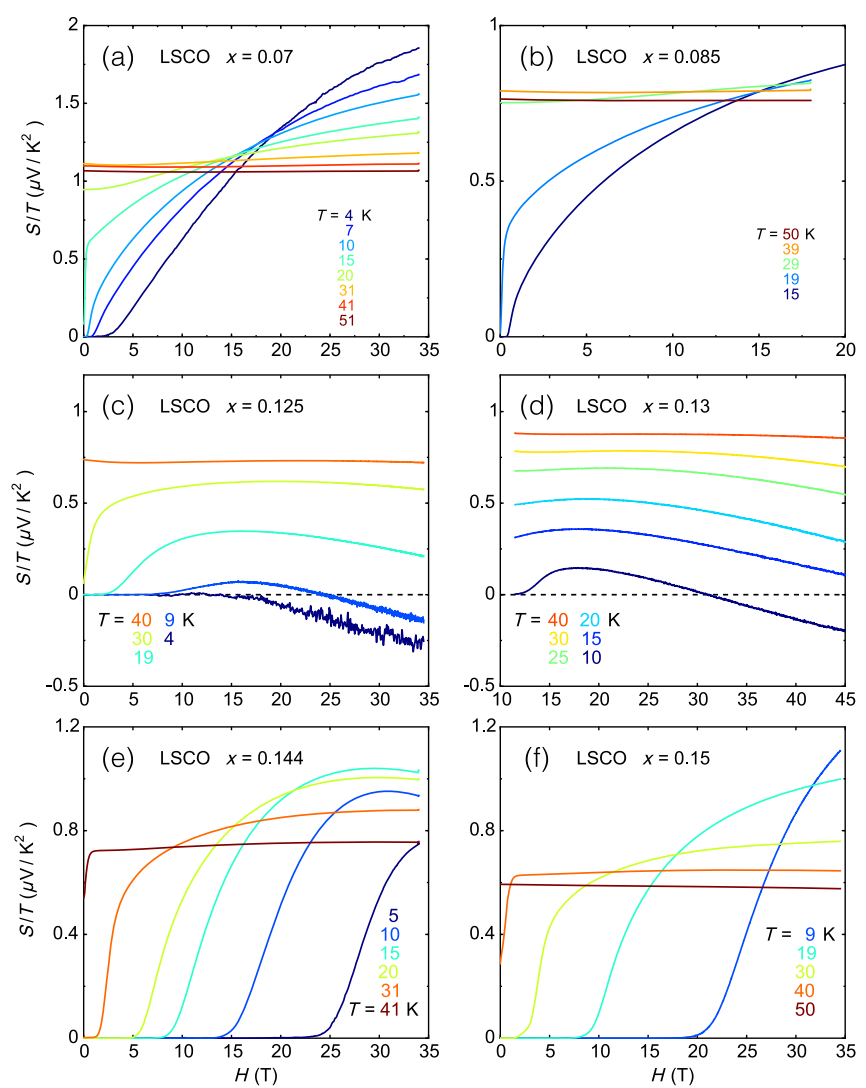

FIG. 2. Isotherms of the Seebeck coefficient in LSCO, plotted as $S / T$ vs magnetic field $H$, at various temperatures, as indicated, for six samples, with $x=0.07$ (a), $x=0.085$ (b), $x=0.125$ (c), $x=0.13(\mathrm{~d}), x=0.144$ (e), and $x=0.15$ (f). For $x=0.125$ and $0.13, S / T$ at high $H$ decreases at low temperatures, to reach negative values. For $x=0.144, S / T$ also decreases at low temperatures, below $15 \mathrm{~K}$. This decrease is the signature of FSR. In contrast, for $x=0.07$ and $0.15, S / T$ at the highest measured field keeps increasing with decreasing temperature down to the lowest temperature. This shows that there is no FSR at those dopings, at least down to $4 \mathrm{~K}$ and $9 \mathrm{~K}$, respectively. The same is true at $x=0.085$, at least down to $15 \mathrm{~K}$.

$T=8 \mathrm{~K}$ is increasingly negative with increasing $H$-inset of Fig. 4(b) - confirming that a negative $S$ is a property of the normal state also at that doping.] The location of the peak in $S / T$ vs $T$ is seen to decrease from $T_{\max }=45 \mathrm{~K}$ at $x=0.12$, to $T_{\max }=42.5 \mathrm{~K}$ at $x=0.125$, to $T_{\max }=40 \mathrm{~K}$ at $x=0.13$. Those $T_{\max }$ values are plotted on the phase diagram of LSCO in Fig. 1(b). Raising the doping further, we observe that $T_{\max }$ continues its steady descent. Indeed, at $p=0.144, S / T$ now peaks at $T_{\max } \simeq 15 \mathrm{~K}$ [Fig. 3(b)]. Extrapolating this trend yields $T_{\max } \rightarrow 0$ at $p \rightarrow 0.15$ [Fig. 1(b)]. Our data at $x=0.15$ confirm this, with $S / T$ showing no decrease down to at least $9 \mathrm{~K}$ [Figs. 2(f) and 3(b)]. This shows that FSR in LSCO ends at a critical doping $p_{\mathrm{FSR}}=0.15 \pm 0.005$.

At $x=0.07$, the normal-state $S / T$ increases monotonically with decreasing $T$, down to our lowest temperature [Fig. 3(a)]. There is clearly no FSR at that doping. At
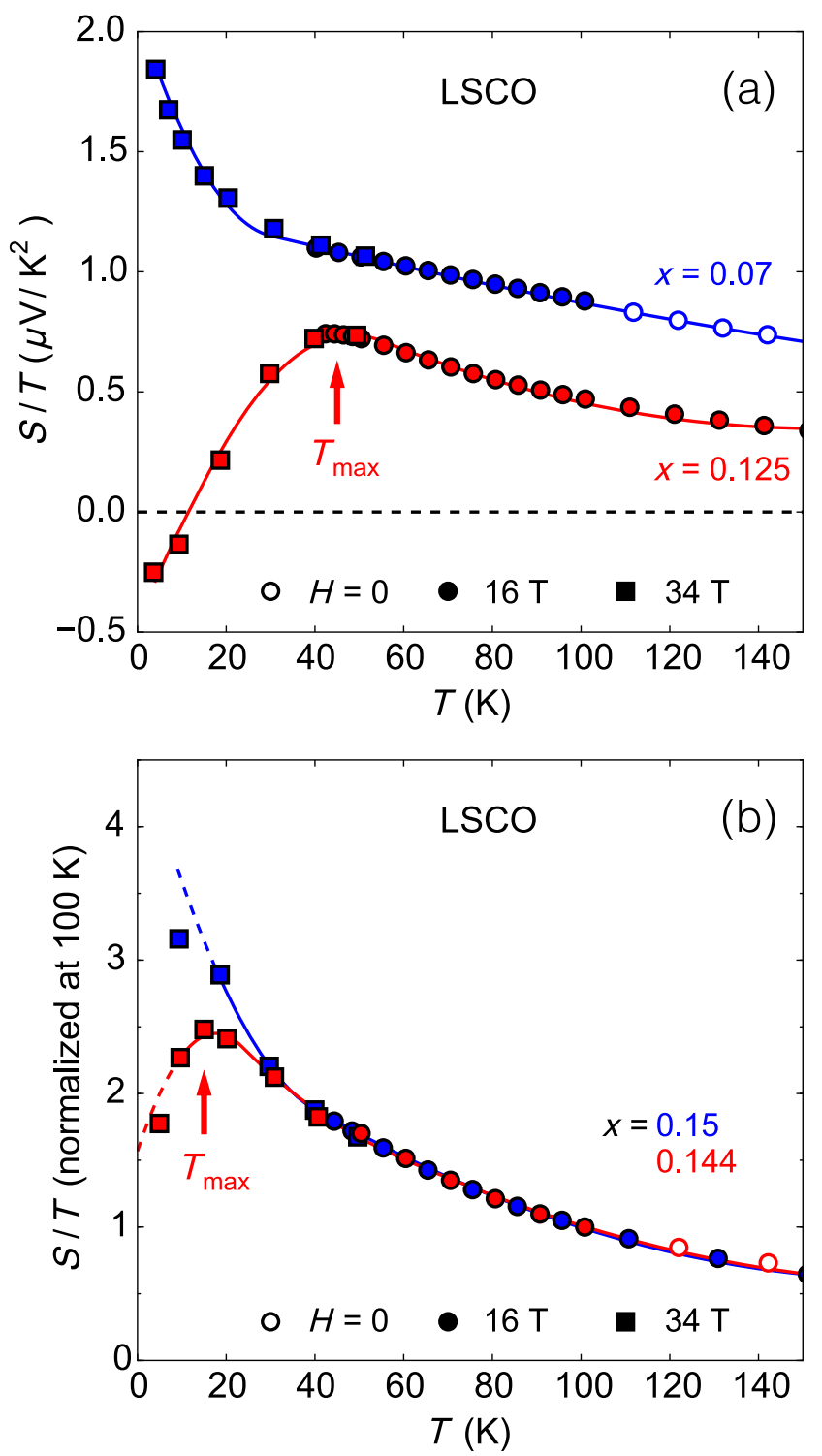

FIG. 3. Seebeck coefficient of LSCO, plotted as $S / T$ vs temperature $T$, measured in a magnetic field $H=0$ (open circles), 16 T (full circles), and $34 \mathrm{~T}$ (squares), for four samples, with $x=0.07$ and 0.125 (a), and $x=0.144$ and 0.15 (b). The data in panel (b) are normalized to their value at $T=100 \mathrm{~K}$. All data points represent the normal state, for which the solid lines are a guide to the eye, except the lowest point for each of $x=0.144$ and $x=0.15$ [panel (b)]. For these two points, the isotherms are still going up the superconducting transition (Fig. 2). The dashed lines are an extension of the normalstate behavior based on extrapolating those isotherms beyond $34 \mathrm{~T}$. $T_{\max }$ marks the temperature below which $S / T$ decreases at low temperatures (arrow), in some cases reaching negative values, as seen here for $x=0.125$. This decrease is the signature of FSR. Note how the data for $x=0.144$ and $x=0.15$ split below $T \simeq 30 \mathrm{~K}$, with the former dropping at low $T$ because of FSR and the latter showing no decrease, and hence no FSR (at least down to $9 \mathrm{~K}$ ).

$x=0.085$, although we only measured up to 18 or $20 \mathrm{~T}$, we observe that $S / T$ at $H=18 \mathrm{~T}$ increases as $T \rightarrow 0$, at least down to $15 \mathrm{~K}$ [Fig. 2(b)]. So here $T_{\max }<15 \mathrm{~K}$. In Fig. 1(b), we plot $T_{\max }$ vs $p$ for our eight samples, with 

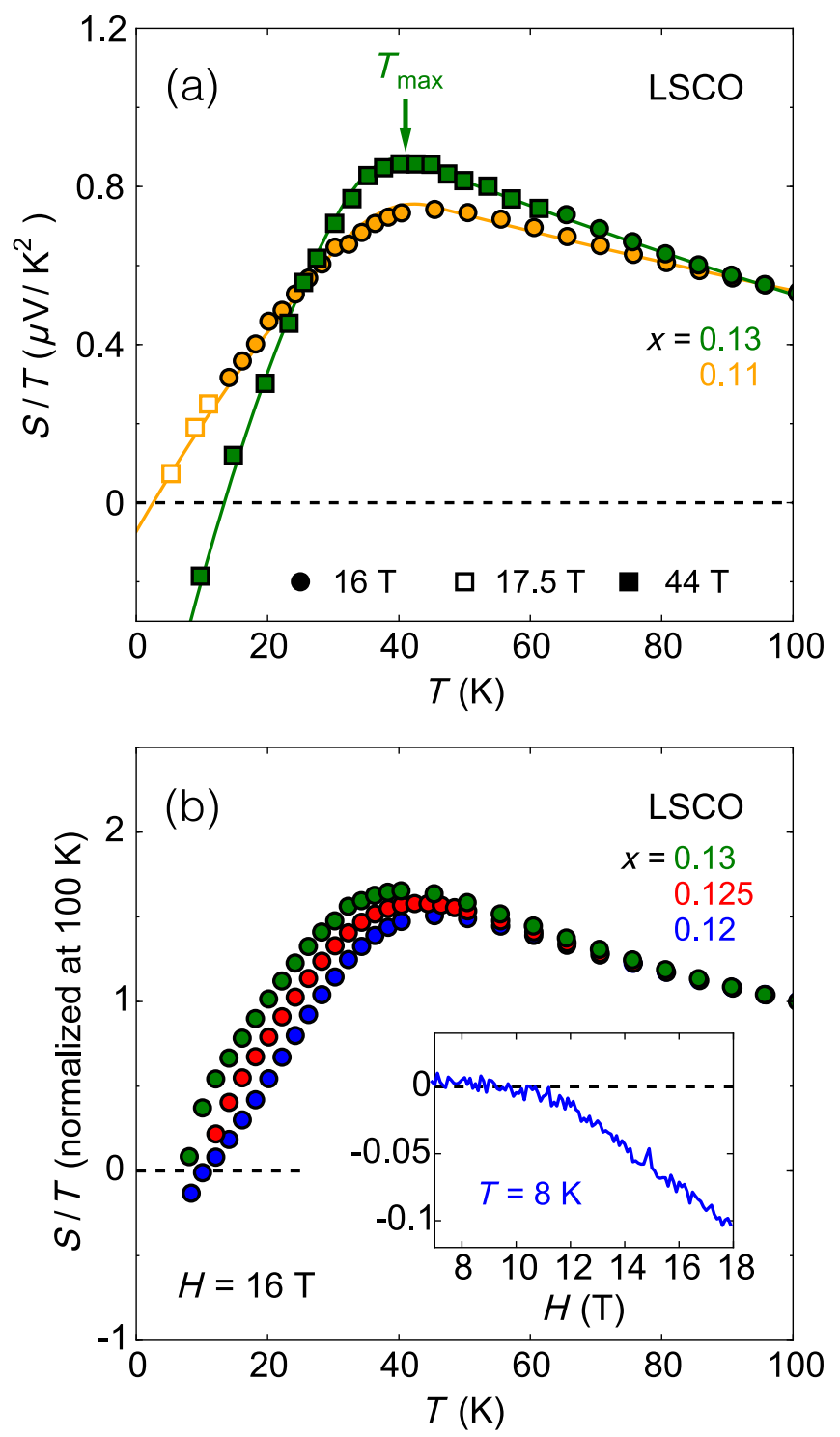

FIG. 4. Same as in Fig. 3, for samples with $x=0.11$ (yellow), $x=0.12$ (blue), $x=0.125$ (red), and $x=0.13$ (green), measured at $H=16 \mathrm{~T}$ (full circles), $17.5 \mathrm{~T}$ (open squares), and $44 \mathrm{~T}$ (full squares). The data in panel (b) are normalized to their value at $T=100 \mathrm{~K}$. FSR is clearly observed in all four samples, as a drop in $S / T$ at low temperature. Inset of panel (b): Isotherm at $T=$ $8 \mathrm{~K}$ for $x=0.12$, showing that $S / T$ becomes increasingly negative with an increasing field, demonstrating that the negative $S$ is a property of the normal state.

their uncertainty, and thereby delineate the region where FSR occurs in the $T-p$ phase diagram of LSCO. We see that the FSR region peaks at $p \simeq 0.12$ and is confined between $p \simeq 0.085$ and $p=p_{\mathrm{FSR}}=0.15 \pm 0.005$.

\section{HALL COEFFICIENT}

In Fig. 5(c), the Hall coefficient of our LSCO crystal with $x=0.12$, measured at $H=16 \mathrm{~T}$, is plotted as $R_{\mathrm{H}}$ vs $T$. We see that $R_{\mathrm{H}}(T)$ drops below $T \simeq 50 \mathrm{~K}$ and becomes negative below $T \simeq 20 \mathrm{~K}$. Data for our crystals with $x=0.11,0.125$, and 0.13 are very similar, also negative at low $T$, and all in excellent agreement with prior low-field data on single crystals of LSCO with $x=0.12$ [27]. (The absence of a negative $R_{\mathrm{H}}$ in previous high-field data on thin films of LSCO [28] may be due to the higher disorder of such samples.) A similar drop in $R_{\mathrm{H}}(T)$ has been seen in Eu-LSCO [29] and in $\mathrm{La}_{1.4-x} \mathrm{Nd}_{0.6} \mathrm{Sr}_{x} \mathrm{CuO}_{4}$ (Nd-LSCO) [30], when $p \simeq 0.12$; in both materials, it is closely linked to the onset of CDW order.

\section{DISCUSSION}

Taken together, the negative Hall and Seebeck coefficients in the normal state of LSCO are conclusive evidence of FSR in this material, in the vicinity of $p=0.12$. This adds up to the previous three cases, namely, YBCO, EuLSCO, and Hg1201. In all four cases, the FSR occurs in a region of the $T-p$ phase diagram where CDW modulations have been detected by XRD (Fig. 1). The link between CDW and FSR is robust.

It is instructive to compare LSCO and YBCO. The two phase diagrams are similar (Fig. 1). In both cases, $T_{\max }$ and $T_{\mathrm{CDW}}$ peak at $p=0.12$, and the region of FSR is confined to similar ranges - from $p \simeq 0.085$ to $p=0.15$ in LSCO and from $p=0.08$ to $p \simeq 0.15$ in YBCO [4]. In Fig. 5, we compare data for LSCO and YBCO directly, at $p=0.12$. The CDW modulations detected by XRD emerge below a temperature twice as high in YBCO compared to LSCO [Fig. 5(a)]: $T_{\mathrm{CDW}} \simeq 150 \mathrm{~K}$ in $\mathrm{YBCO}$ vs $T_{\mathrm{CDW}} \simeq 75 \mathrm{~K}$ in LSCO. Correspondingly, the FSR is detected at a temperature twice as high in YBCO compared to LSCO, with $T_{\text {max }} \simeq 100 \mathrm{~K}$ in $\mathrm{YBCO}$ vs $T_{\max } \simeq 50 \mathrm{~K}$ in $\mathrm{LSCO}$ [Fig. 5(b)]. All this suggests that CDW ordering is a stronger tendency in YBCO than in LSCO. Intriguingly, the superconducting transition temperature $T_{\mathrm{c}}$ is roughly twice as high in YBCO as compared to LSCO [see cusp in Fig. 5(a)]. This raises the interesting possibility that the same underlying mechanism, perhaps magnetic, fuels both superconductivity and CDW order [31].

Given that FSR in LSCO ends at $p_{\mathrm{FSR}}=0.15$, we infer that this is also where CDW order ends. This is consistent with recent XRD measurements that detect no $\mathrm{CDW}$ modulations in LSCO at $x=0.15$ [32]. (The same consistency is observed at $x=0.085$, where again no CDW modulations are detected by XRD [32].) We thus arrive at key information: The CDW phase in LSCO ends at the critical doping $p_{\mathrm{CDW}}=0.15$.

This is distinctly below the critical point where the pseudogap phase is believed to end in LSCO, at $p^{\star} \simeq 0.19$, as determined from the normal-state resistivity measured in high magnetic fields [26]. This clear separation reveals that the pseudogap phase is not caused by the CDW ordering. Instead, it suggests that CDW order is a secondary instability of the pseudogap phase. A very similar separation was recently observed in YBCO from high-field Hall 

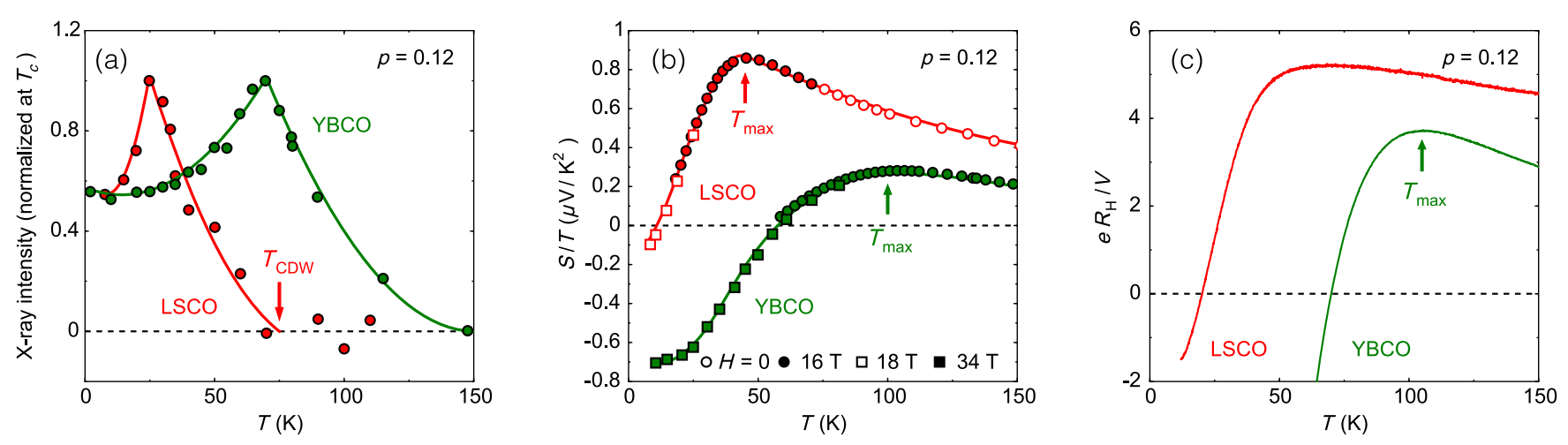

FIG. 5. Comparison of LSCO (red) and YBCO (green) at $p=0.12$. (a) Temperature dependence of the X-ray intensity associated with the CDW modulations, normalized at $T_{\mathrm{c}}$, detected in LSCO [13] and YBCO [10]. Lines are a guide to the eye. The cusp is at $T_{\mathrm{c}}$. (b) Normal-state Seebeck coefficient of LSCO (this work) and YBCO [6], measured in a magnetic field as indicated, plotted as $S / T$ vs $T$. $T_{\max }$ is the temperature below which $S / T$ drops to reach negative values at low temperatures (arrow), the signature of FSR. This $T_{\max }$ is plotted as full circles in Fig. 1. Lines are a guide to the eye. (c) Hall coefficient of LSCO at $H=16 \mathrm{~T}$ and YBCO at $H=15 \mathrm{~T}$ [2], plotted as $e R_{\mathrm{H}} / V$, where $e$ is the electron charge and $V$ the volume per planar $\mathrm{Cu}$ atom. $T_{\max }$ is the temperature below which $R_{\mathrm{H}}(T)$ drops to reach negative values at low temperatures (arrow), another signature of FSR. $T_{\max }$ is plotted as open circles in Fig. 1(a) [4].

effect measurements, with $p_{\mathrm{CDW}}=0.16 \pm 0.005$ and $p^{\star} \simeq 0.19$ [33]. This strongly suggests that a separation of $p_{\mathrm{CDW}}$ and $p^{\star}$ is a generic property of cuprates.

\section{SUMMARY}

Our high-field measurements of the Seebeck coefficient in the cuprate superconductor LSCO reveal that its normalstate Fermi surface undergoes a reconstruction at low temperatures, in the doping range $0.085<p<0.15$. In analogy with the cuprates YBCO, Eu-LSCO, and Hg1201, we attribute this FSR to the CDW modulations detected by $\mathrm{XRD}$ in the very same doping range. Combined with XRD data on LSCO, our Seebeck data make a compelling case that $\mathrm{CDW}$ modulations disappear at $p=p_{\mathrm{CDW}}=0.15$, so the field-induced nonsuperconducting ground state of LSCO above $p=0.15$ has no CDW order. Because the pseudogap phase in the normal state of LSCO extends up to $p \simeq 0.19$, we infer that the pseudogap is not tied to CDW ordering. Instead, the CDW modulations appear to be a secondary instability of the pseudogap phase.

\section{ACKNOWLEDGMENTS}

We thank J. Chang, N. E. Hussey, M.-H. Julien, P. A. Lee, B. J. Ramshaw, S. Sachdev, J. L. Tallon, and G. A. Sawatzky for stimulating discussions. A portion of this work was performed at the National High Magnetic Field Laboratory, which is supported by the National Science Foundation Cooperative Agreement No. DMR-1157490, the State of Florida, and the U.S. Department of Energy. Another portion of this work was performed at the Laboratoire National des Champs Magnétiques Intenses of the CNRS, member of the European Magnetic Field Laboratory. L. T. acknowledges support from the Canadian Institute for Advanced Research (CIFAR) and funding from the National Science and Engineering Research Council of
Canada (NSERC), the Fonds de recherche du QuébecNature et Technologies (FRQNT), the Canada Foundation for Innovation (CFI), and a Canada Research Chair.

[1] N. Doiron-Leyraud, C. Proust, D. LeBoeuf, J. Levallois, J.-B. Bonnemaison, R. Liang, D. A. Bonn, W. N. Hardy, and L. Taillefer, Quantum Oscillations and the Fermi Surface in an Underdoped High- $T_{c}$ Superconductor, Nature (London) 447, 565 (2007).

[2] D. LeBoeuf, N. Doiron-Leyraud, J. Levallois, R. Daou, J.-B. Bonnemaison, N. E. Hussey, L. Balicas, B. J. Ramshaw, R. Liang, D. A. Bonn et al., Electron Pockets in the Fermi Surface of Hole-Doped High- $T_{c}$ Superconductors, Nature (London) 450, 533 (2007).

[3] L. Taillefer, Fermi Surface Reconstruction in High- $T_{c}$ Superconductors, J. Phys. Condens. Matter 21, 164212 (2009).

[4] D. LeBoeuf, N. Doiron-Leyraud, B. Vignolle, M. Sutherland, B. J. Ramshaw, J. Levallois, R. Daou, F. Laliberté, O. Cyr-Choinière, J. Chang et al., Lifshitz Critical Point in the Cuprate Superconductor $\mathrm{YBa}_{2} \mathrm{Cu}_{3} \mathrm{O}_{y}$ from High-Field Hall Effect Measurements, Phys. Rev. B 83, 054506 (2011).

[5] J. Chang, R. Daou, C. Proust, D. LeBoeuf, N. DoironLeyraud, F. Laliberté, B. Pingault, B. J. Ramshaw, R. Liang, D. A. Bonn et al., Nernst and Seebeck Coefficients of the Cuprate Superconductor $\mathrm{YBa}_{2} \mathrm{Cu}_{3} \mathrm{O}_{6.67}$ : A Study of Fermi Surface Reconstruction, Phys. Rev. Lett. 104, 057005 (2010).

[6] F. Laliberté, J. Chang, N. Doiron-Leyraud, E. Hassinger, R. Daou, M. Rondeau, B. J. Ramshaw, R. Liang, D. A. Bonn, W. N. Hardy et al., Fermi-Surface Reconstruction by Stripe Order in Cuprate Superconductors, Nat. Commun. 2, 432 (2011).

[7] J. Fink, V. Soltwisch, J. Geck, E. Schierle, E. Weschke, and B. Buchner, Phase Diagram of Charge Order in $\mathrm{La}_{1.8-x} \mathrm{Eu}_{0.2} \mathrm{Sr}_{x} \mathrm{CuO}_{4}$ from Resonant Soft X-Ray Diffraction, Phys. Rev. B 83, 092503 (2011). 
[8] T. Wu, H. Mayaffre, S. Krämer, M. Horvatić, C. Berthier, W. N. Hardy, R. Liang, D. A. Bonn, and M.-H. Julien, Magnetic-Field-Induced Charge-Stripe Order in the High-Temperature Superconductor $\mathrm{YBa}_{2} \mathrm{Cu}_{3} \mathrm{O}_{y}$, Nature (London) 477, 191 (2011).

[9] G. Ghiringhelli, M. Le Tacon, M. Minola, S. BlancoCanosa, C. Mazzoli, N. B. Brookes, G. M. De Luca, A. Frano, D. G. Hawthorn, F. He et al., Long-Range Incommensurate Charge Fluctuations in $(\mathrm{Y}, \mathrm{Nd}) \mathrm{Ba}_{2} \mathrm{Cu}_{3} \mathrm{O}_{6+x}$, Science 337, 821 (2012).

[10] J. Chang, E. Blackburn, A. T. Holmes, N. B. Christensen, J. Larsen, J. Mesot, R. Liang, D. A. Bonn, W. N. Hardy, A. Watenphul et al., Direct Observation of Competition between Superconductivity and Charge Density Wave Order in $\mathrm{YBa}_{2} \mathrm{Cu}_{3} \mathrm{O}_{6.67}$, Nat. Phys. 8, 871 (2012).

[11] M. Hücker, N. B. Christensen, A. T. Holmes, E. Blackburn, E. M. Forgan, R. Liang, D. A. Bonn, W. N. Hardy, O. Gutowski, M. v. Zimmermann, S. M. Hayden, and J. Chang, Competing Charge, Spin, and Superconducting Orders in Underdoped $\mathrm{YBa}_{2} \mathrm{Cu}_{3} \mathrm{O}_{y}$, Phys. Rev. B 90, 054514 (2014).

[12] S. Blanco-Canosa, A. Frano, E. Schierle, J. Porras, T. Loew, M. Minola, M. Bluschke, E. Weschke, B. Keimer, and M. Le Tacon, Resonant X-Ray Scattering Study of ChargeDensity Wave Correlations in $\mathrm{YBa}_{2} \mathrm{Cu}_{3} \mathrm{O}_{6+x}$, Phys. Rev. $\mathrm{B}$ 90, 054513 (2014).

[13] T. P. Croft, C. Lester, M. S. Senn, A. Bombardi, and S. M. Hayden, Charge Density Wave Fluctuations in $\mathrm{La}_{2-x} \mathrm{Sr}_{x} \mathrm{CuO}_{4}$ and Their Competition with Superconductivity, Phys. Rev. B 89, 224513 (2014).

[14] N. B. Christensen, J. Chang, J. Larsen, M. Fujita, M. Oda, M. Ido, N. Momono, E. M. Forgan, A. T. Holmes, J. Mesot, M. Huecker, and M. v. Zimmermann, Bulk Charge Stripe Order Competing with Superconductivity in $\mathrm{La}_{2-x} \mathrm{Sr}_{x} \mathrm{CuO}_{4}$ $(\mathrm{x}=0.12)$, arXiv:1404.3192.

[15] D. Haug, V. Hinkov, Y. Sidis, P. Bourges, N. B. Christensen, A. Ivanov, T. Keller, C. T. Lin, and B. Keimer, Neutron Scattering Study of the Magnetic Phase Diagram of Underdoped $\mathrm{YBa}_{2} \mathrm{Cu}_{3} \mathrm{O}_{6+x}$, New J. Phys. 12, 105006 (2010).

[16] J. Chang, Ch. Niedermayer, R. Gilardi, N. B. Christensen, H. M. Ronnow, D. F. McMorrow, M. Ay, J. Stahn, O. Sobolev, A. Hiess et al., Tuning Competing Orders in $\mathrm{La}_{2-x} \mathrm{Sr}_{x} \mathrm{CuO}_{4}$ Cuprate Superconductors by the Application of an External Magnetic Field, Phys. Rev. B 78, 104525 (2008).

[17] M. Kofu, S.-H. Lee, M. Fujita, H.-J. Kang, H. Eisaki, and K. Yamada, Hidden Quantum Spin-Gap State in the Static Stripe Phase of High-Temperature $\mathrm{La}_{2-x} \mathrm{Sr}_{x} \mathrm{CuO}_{4}$ Superconductors, Phys. Rev. Lett. 102, 047001 (2009).

[18] S. Wakimoto, G. Shirane, Y. Endoh, K. Hirota, S. Ueki, K. Yamada, R. J. Birgeneau, M. A. Kastner, Y. S. Lee, P. M. Gehring, and S. H. Lee, Observation of Incommensurate Magnetic Correlations at the Lower Critical Concentration for Superconductivity in $\mathrm{La}_{2-x} \mathrm{Sr}_{x} \mathrm{CuO}_{4}(\mathrm{x}=0.05)$, Phys. Rev. B 60, R769 (1999).

[19] B. Lake, H. M. Rønnow, N. B. Christensen, G. Aeppli, K. Lefmann, D. F. McMorrow, P. Vorderwisch, P. Smeibidl, N. Mangkorntong, T. Sasagawa et al., Antiferromagnetic Order Induced by an Applied Magnetic Field in a HighTemperature Superconductor, Nature (London) 415, 299 (2002).
[20] H. Kimura, K. Hirota, H. Matsushita, K. Yamada, Y. Endoh, S-H. Lee, C. F. Majkrzak, R. Erwin, G. Shirane, M. Greven et al., Neutron-Scattering Study of Static Antiferromagnetic Correlations in $\mathrm{La}_{2-x} \mathrm{Sr}_{x} \mathrm{Cu}_{1-y} \mathrm{Zn}_{y} \mathrm{O}_{4}$, Phys. Rev. B 59, 6517 (1999).

[21] T. Wu, H. Mayaffre, S. Krämer, M. Horvatić, C. Berthier, W. N. Hardy, R. Liang, D. A. Bonn, and M-H. Julien, Incipient Charge Order Observed by NMR in the Normal State of $\mathrm{YBa}_{2} \mathrm{Cu}_{3} \mathrm{O}_{y}$, Nat. Commun. 6, 6438 (2015).

[22] N. Doiron-Leyraud, S. Lepault, O. Cyr-Choinière, B. Vignolle, G. Grissonnanche, F. Laliberté, J. Chang, N. Barišić, M. K. Chan, L. Ji et al., Hall, Seebeck, and Nernst Coefficients of Underdoped $\mathrm{HgBa}_{2} \mathrm{CuO}_{4+\delta}$ : Fermi-Surface Reconstruction in an Archetypal Cuprate Superconductor, Phys. Rev. X 3, 021019 (2013).

[23] N. Barišić, S. Badoux, M. K. Chan, C. Dorow, W. Tabis, B. Vignolle, G. Yu, J. Béard, X. Zhao, C. Proust, and M. Greven, Universal Quantum Oscillations in the Underdoped Cuprate Superconductors, Nat. Phys. 9, 761 (2013).

[24] W. Tabis, Y. Li, M. Le Tacon, L. Braicovich, A. Kreyssig, M. Minola, G. Dellea, E. Weschke, M. J. Veit, M. Ramazanoglu et al., Charge Order and Its Connection with Fermi-Liquid Charge Transport in a Pristine High- $T_{c}$ Cuprate, Nat. Commun. 5, 5875 (2014).

[25] V. Thampy, M. P. M. Dean, N. B. Christensen, L. Steinke, Z. Islam, M. Oda, M. Ido, N. Momono, S. B. Wilkins, and J. P. Hill, Rotated Stripe Order and Its Competition with Superconductivity in $\mathrm{La}_{1.88} \mathrm{Sr}_{0.12} \mathrm{CuO}_{4}$, Phys. Rev. B 90, 100510 (2014).

[26] R. A. Cooper, Y. Wang, B. Vignolle, O. J. Lipscombe, S. M. Hayden, Y. Tanabe, T. Adachi, Y. Koike, M. Nohara, H. Takagi, C. Proust, and N. E. Hussey, Anomalous Criticality in the Electrical Resistivity of $\mathrm{La}_{2-x} \mathrm{Sr}_{x} \mathrm{CuO}_{4}$, Science 323, 603 (2009).

[27] T. Suzuki, T. Goto, K. Chiba, M. Minami, Y. Oshima, T. Fukase, M. Fujita, and K. Yamada, Hall Coefficient of $\mathrm{La}_{1.88-y} \mathrm{Y}_{y} \mathrm{Sr}_{0.12} \mathrm{CuO}_{4}(\mathrm{y}=0,0.04)$ at Low Temperatures under High Magnetic Fields, Phys. Rev. B 66, 104528 (2002).

[28] F. F. Balakirev, J. B. Betts, A. Migliori, I. Tsukada, Y. Ando, and G.S. Boebinger, Quantum Phase Transition in the Magnetic-Field-Induced Normal State of Optimum-Doped High- $T_{c}$ Cuprate Superconductors at Low Temperatures, Phys. Rev. Lett. 102, 017004 (2009).

[29] O. Cyr-Choinière, R. Daou, F. Laliberté, D. LeBoeuf, N. Doiron-Leyraud, J. Chang, J.-Q. Yan, J.-G. Cheng, J.-S. Zhou, J. B. Goodenough et al., Enhancement of the Nernst Effect by Stripe Order in a High- $T_{c}$ Superconductor, Nature (London) 458, 743 (2009).

[30] T. Noda, H. Eisaki, and S. Uchida, Evidence for OneDimensional Charge Transport in $\mathrm{La}_{2-x-y} \mathrm{Nd}_{y} \mathrm{Sr}_{x} \mathrm{CuO}_{4}$, Science 286, 265 (1999).

[31] K. B. Efetov, H. Meier, and C. Pépin, Pseudogap State Near a Quantum Critical Point, Nat. Phys. 9, 442 (2013).

[32] T. P. Croft and S. M. Hayden (private communication).

[33] S. Badoux, W. Tabis, F. Laliberté, G. Grissonnanche, B. Vignolle, D. Vignolles, J. Béard, D. A. Bonn, W. N. Hardy, R. Liang et al., Change of Carrier Density at the Pseudogap Critical Point of a Cuprate Superconductor, Nature (London) 531, 210 (2016). 